\title{
La toma del agua zacatecana
}

\author{
Darcy Tetreault*
}

Los acuíferos que abastecen la Zona Conurbada de Zacatecas y Guadalupe (ZCZG) están sobreexplotados, contaminados y pobremente regulados. Existe una crisis y la gran mayoría de la población local no tiene conciencia de ello. Durante más de un cuarto de siglo, las agencias gubernamentales encargadas de administrar el agua han mostrado incapacidad para encontrar soluciones efectivas. No pueden. Están subordinadas al capital y su imperativo de acumulación bajo el actual modelo neoliberal.

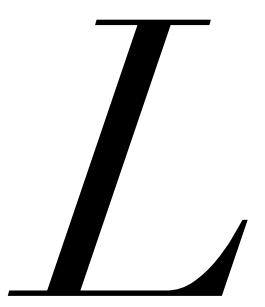

A ZONA Conurbada de Zacatecas y Guadalupe (ZCZG) se ubica en un altiplano árido y templado, al este de la Sierra Madre Occidental, en el centro norte del país. En esta región la precipitación media anual es de sólo $450 \mathrm{~mm}$ y las aguas superficiales son escasas y fuertemente contaminadas. Por tanto, se explotan los acuíferos locales Benito Juárez, Calera y Guadalupe-Bañuelos para abastecer las necesidades de la población urbana.

* Docente-investigador de la Unidad Académica en Estudios del Desarrollo de la Universidad Autónoma de Zacatecas, México.
Los mismos acuíferos se explotan para potenciar las actividades agrícolas e industriales de la región. Con todo, están sobreexplotados. Además, están contaminados con metales pesados, lo que representa un serio riesgo para la salud de los pobladores de la ZCZG.

En este texto se analizan las diversas dimensiones de la problemática del agua en la ZCZG con el argumento de que, en conjunto, constituyen una crisis y un conflicto socioambiental latente. Además, se presentan los resultados de una encuesta que fue aplicada en 2013 para medir los niveles de conciencia sobre esta crisis. Como se verá, en general los pobladores locales no están enterados de la problemática. Las agencias gubernamentales encargadas de administrar el agua en la ZCZG han mostrado incapacidad de enfrentar la 
situación crítica con políticas públicas efectivas. Esto se deriva de la subordinación a los imperativos del actual modelo de desarrollo industrial neoliberal.

\section{SOBREEXPLOTACión de ACUífEROS}

Y FALTA DE CONTABILIDAD

En un documento fechado en I988, la ahora difunta Secretaría de Agricultura y Recursos Hidráulicos (SARH) hace constar que "la Ciudad de Zacatecas enfrenta ciertos problemas con el abastecimiento de agua, ya que las fuentes actuales presentan abatimiento de $.5 \mathrm{~m}$ anuales". ' Así, las autoridades en materia de agua han estado conscientes de este problema durante, por lo menos, 25 años.

En rg89 se creó la Comisión Nacional del Agua (Conagua) con la misión de "administrar y preservar las aguas nacionales y sus bienes inherentes, para lograr su uso sustentable, con la corresponsabilidad de los tres órdenes de gobierno y la sociedad en general". ${ }^{2}$ Tres años más tarde, se promulgó la Ley de Aguas Nacionales (LAN), en donde el líquido vital se considera un bien económico y se sientan las bases para crear mercados para la transferencia de derechos de usufructo en los distritos de riego. La LAN confiere a la Conagua la responsabilidad de publicar en el Diario Oficial de la Federación la disponibilidad de aguas subterráneas en cada acuífero.

En r992, mismo año en que se publicó la LAN, Conagua actualizó el Censo de Aprovechamientos para calcular la disponibilidad de agua en los acuíferos que abastecen la ZCZG, con base en estudios técnicos sobre la extracción consignada. De este modo, se calculó que dos de ellos estaban sobreexplotados: Benito Juárez con un déficit de 2.9 millones de metros cúbicos de agua por año $\left(\mathrm{Mm}^{3} /\right.$ año $)$ y Calera con un enorme déficit de 4I.r Mm³/año. En r992, el acuífero Guadalupe-Bañuelos todavía tenía disponibles 2.0 $\mathrm{Mm}^{3} / \mathrm{año} \mathrm{en} 199^{2} .^{3}$

No se ha actualizado el Censo de Aprovechamientos desde entonces. Los ingenieros de la Conagua realmente no saben cuál es el actual grado de sobreexplotación de los acuíferos. Los cálculos más recientes se basan en los volúmenes de concesión, los cuales superan las recargas naturales de los tres acuíferos, dando lugar a déficits de I.ı4 $\mathrm{Mm}^{3}$ /año, $67.75 \mathrm{Mm}^{3} /$ año y ı. $95 \mathrm{Mm}^{3} /$ año, respetivamente. ${ }^{4}$ Cabe enfatizar, sin embargo, que estos cálculos pueden estar lejos de la realidad, puesto que $89 \%$ de los usuarios que tienen concesiones para extraer agua de estos tres acuíferos no cuentan con medidor, a pesar de ser obligatorio, según la ley. ${ }^{5} \mathrm{Ni}$ hablar de los pozos clandestinos.

\section{Contaminación de aguas}

Otro problema es la contaminación. Existen varias fuentes de contaminación de las aguas superficiales y subterráneas en la región de Zacatecas y Guadalupe. En primer lugar, los más de 400 años de minería capitalista en la región han dejado atrás jales y montones de escombros (terreros) que emiten sustancias tóxicas al medio ambiente. Expuestas a la intemperie y a través de los flujos hidrológicos, estas sustancias han sido diseminadas por los valles agrícolas en los alrededores de la capital del estado. ${ }^{6}$

En el municipio de Guadalupe, los residuos minerales tóxicos se han acumulado en la presa La Zacatecana, conocida como "El Pedernalillo", ubicada en el municipio de Guadalupe. La acumulación de estos residuos es tanto que, durante los años ochenta del siglo pasado, varias microempresas se instalaron alrededor de La Zacatecana para extraer plata de los materiales aluviales. Al emplear el mercurio en el proceso de beneficio, estas empresas contribuyeron a exacerbar el problema de contaminación tóxica en el ámbito local.

En un estudio llevado a cabo en 1995 por la compañía Plata Panamericana S. A. de C. v., se detectaron concentraciones de mercurio, plomo, arsénico y bario muy por encima de las concentraciones máximas permisibles por la Agencia de Protección Ambiental de Estados Unidos (EPA, por sus siglas en inglés), en muestras de agua tomadas de seis sitios en la zona de la presa. ${ }^{7}$

Paralelamente, entre i994 y 1996, la Conagua llevó a cabo estudios para examinar la posible existencia de elementos nocivos para la salud en el agua que se suministra a la ZCZG. Se detectaron metales pesados como fierro, plomo, cadmio, mercurio y níquel en concentraciones por arriba de la Norma Oficial Mexicana (NOM). ${ }^{8}$ Es bien conocido que muchos de los metales pesados son cancerígenos.

El mismo informe presta mucha atención a la detección de flúor y arsénico en concentraciones por encima de la Nom en el agua extraída de los acuíferos Benito Juárez y Guadalupe-Bañuelos. Cabe señalar que la ingestión crónica de flúor puede causar fluoro- 


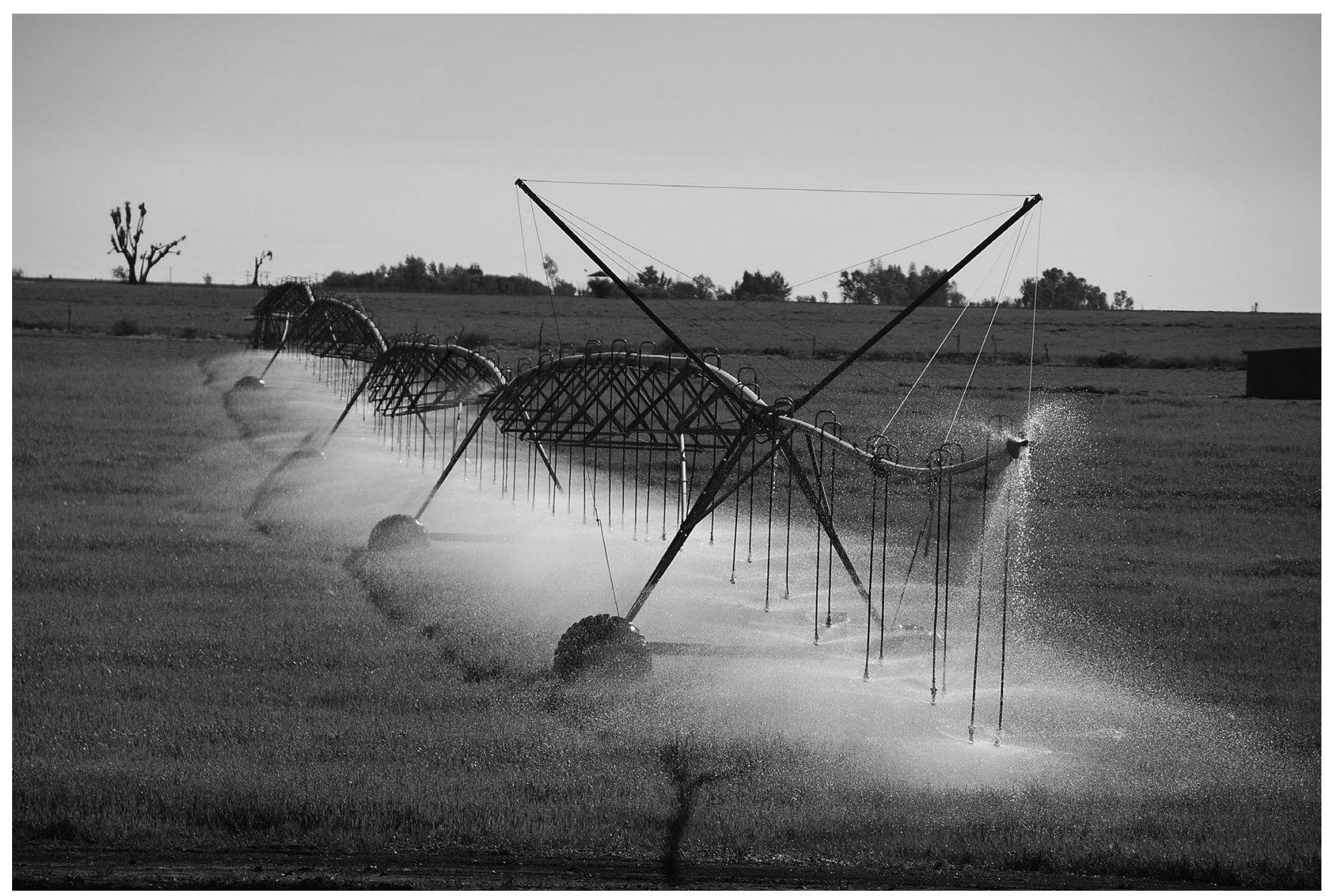

El uso industrial, minero y agrícola del agua contribuyen a la sobrexplotación de los acuiferos, además de las fugas del sistema de distribución de agua destinada al consumo en los hogares.

Foto: Jorge Vázquez.

sis dental y esqueletal, afecta a los riñones, aumenta el riesgo de desarrollar enfermedades de prevalencia carcinogénica y puede perjudicar el desarrollo mental de los niños. 9 Asimismo, el consumo habitual del agua contaminada con arsénico puede causar cáncer de la piel, efectos neurológicos, hipertensión, diabetes y enfermedades cardiovasculares, respiratorias y vasculares periféricas. ${ }^{10}$

Otra fuente de contaminación de las aguas superficiales y subterráneas en el ámbito local son las aguas residuales no tratadas de la ZCZG. Hasta finales de la primera década del siglo XXI, casi todas las aguas negras de Zacatecas y Guadalupe fueron vertidas sin tratamiento alguno en los arroyos que pasan por la zona conurbada. Durante los últimos cuatro años, se han construido tres plantas de tratamiento de aguas residuales (PTAR) en la zona, lo que refleja una tendencia a nivel nacional de construir tales plantas con la participación del sector privado.

En Zacatecas, la agencia gubernamental encargada de manejar las aguas residuales era la Comi- sión Estatal de Agua Potable y Alcantarillado (CEAPA), recientemente reemplazada por la Secretaría del Agua y Medio Ambiente (SAMA). Estos organismos gubernamentales manejan sus cuentas con cierta oscuridad. El exdirector de la CEAPA y de la SAMA, Rafael Gutiérrez Martínez, asegura que más de $9^{\circ}$ por ciento de las obras gestionadas por esta agencia gubernamental fueron a través de licitaciones públicas nacionales. Sin embargo, no ha rendido cuentas a la ciudadanía con respecto a los procesos de licitación, las empresas involucradas y las condiciones de contratación. ${ }^{\text {II }}$

En todo caso, bajo la administración de la CEAPA, en junio de 20 oro se inauguró la Planta Orito en el municipio de Zacatecas, para tratar i 3 por ciento de las aguas residuales de la zona conurbada. En marzo de 2012 fue inaugurada la Planta Poniente en la comunidad de Noria de Gringos, municipio de Morelos, destinada a tratar I7 por ciento; y en septiembre de 2013 se inauguró la planta Osiris al oriente de la ciudad de Guadalupe, supuestamente para tratar el 70 por ciento restante de 


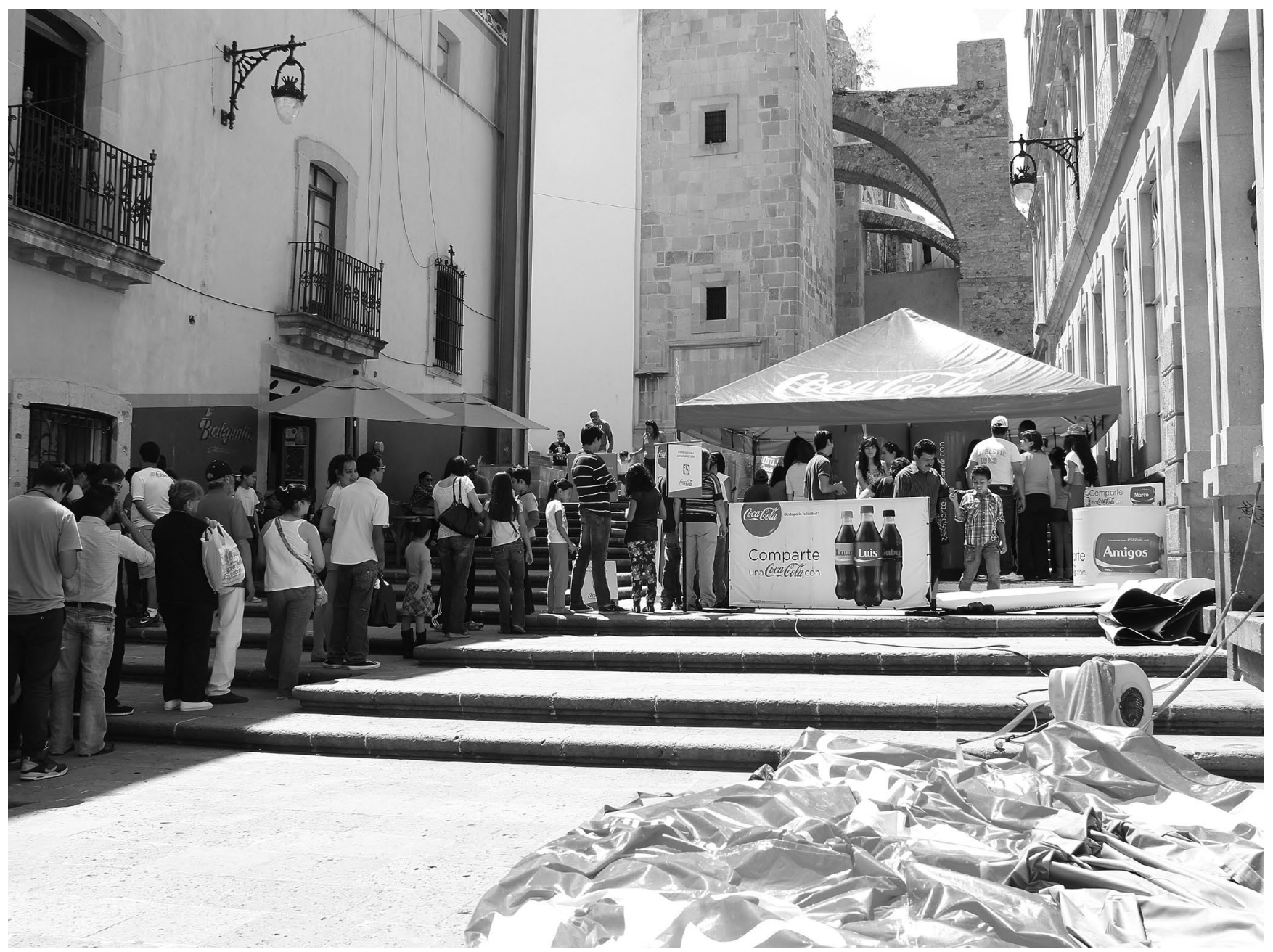

Una larga fila de personas aguarda en una soleada tarde dominical para recibir una lata de bebida edulcorada y carbonatada, que a su vez es una estratagema empresarial para formar fieles consumidores.

Foto: Нumberto Márouez.

dichas aguas. No obstante, hasta mediados de 20I4, esta última planta sólo estaba operando a una sexta parte de su capacidad, lo que quiere decir que la ZCZG continúa expulsando al medio ambiente alrededor de 5 oo litros por segundo de aguas residuales domésticas e industriales no tratadas. Cabe señalar que el contrato para construir esta última PTAR fue asignado al empresario Juan Antonio Rosales Torres, afiliado a la Cámara Mexicana de la Industria de la Construcción (CMIC).

Vale la pena mencionar dos fuentes adicionales de contaminación del agua en la zCZG: la lixiviación de los basureros situados en la periferia de la ciudad y el escurrimiento de los agroquímicos aplicados en la producción agrícola de monocultivos en la región circundante. Parece que estas fuentes de contaminación no han sido estudiadas sistemáticamente hasta la fecha. No obstante, de acuerdo con la Conagua, "pueden estar contribuyendo de manera significativa a incremen- tar el contenido de elementos nocivos para la salud que degradan la calidad del agua". ${ }^{2}$

\section{IRRIGACIÓN}

Como se mencionó en la introducción, los tres acuíferos que abastecen la ZCZG con el líquido vital también son explotados para proveer agua a los agricultores e industrias alrededor de la zona urbana. De esta manera, el agua toma la forma de un bien económico o "insumo" para las actividades productivas de la región, las cuales están orientadas en mayor medida a los mercados nacionales e internacionales.

Como se puede observar en el cuadro i, los volúmenes de agua que se destinan al sector agrícola son mucho mayores que los que se destinan al uso público urbano. Según la distribución de concesiones, 73 por 


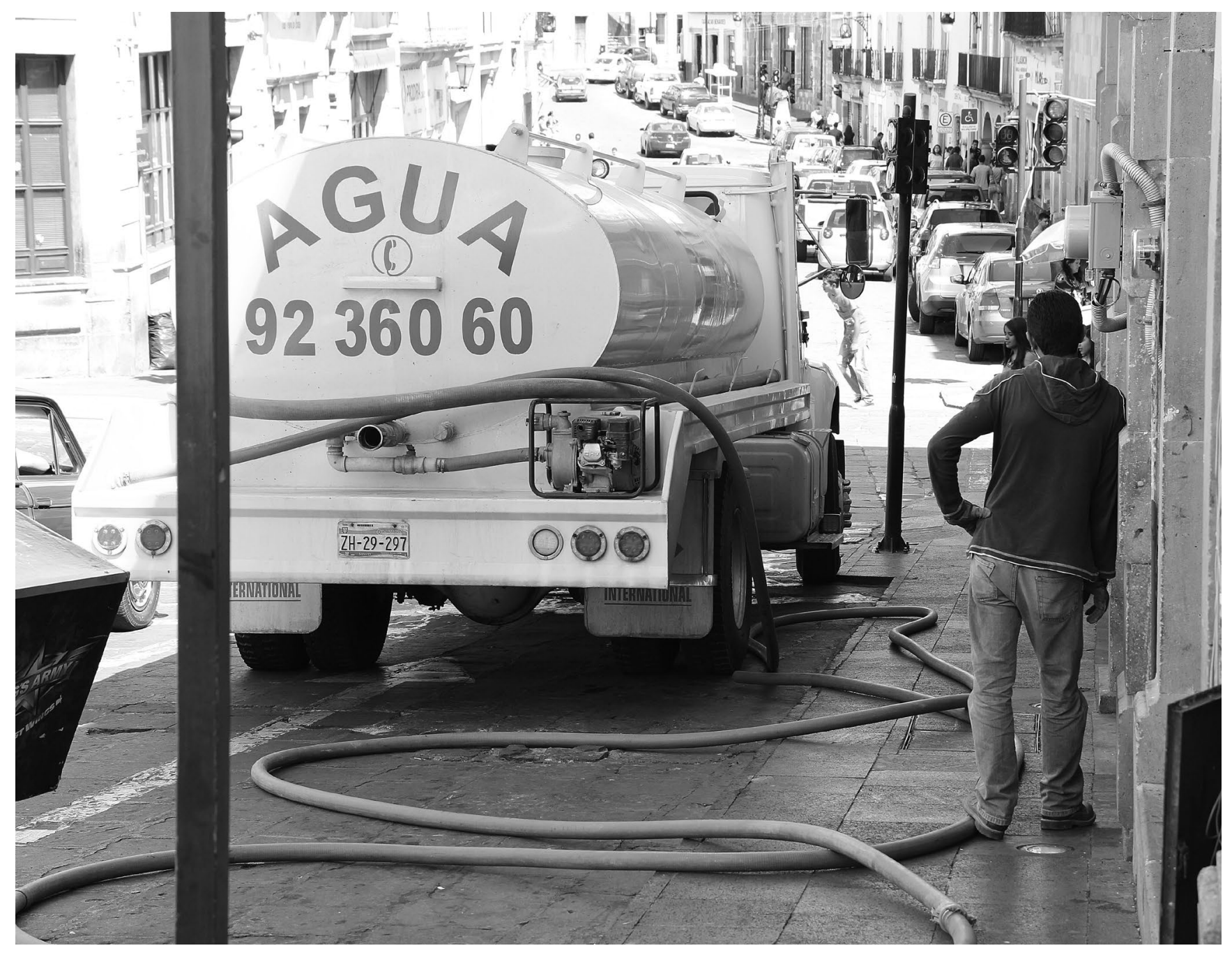

La escasez del suministro de agua en el centro de la ciudad de Zacatecas afecta a las viviendas

y a los establecimientos comerciales que tienen que abastecerse con pipas.

Foto: Humberto Márquez.

ciento del agua que se extrae de los acuíferos Calera, Benito Juárez y Guadalupe-Bañuelos se utiliza para irrigación de cultivos.

Las actividades agrícolas se desarrollan en las llanuras que se encuentran alrededor de la ZCZG, donde se produce frijol, maíz, avena, durazno, ajo, chile y otros cultivos, en menor medida. Desde luego, en una zona semidesértica como la zacatecana, la irrigación es clave. Más de una cuarta parte de las unidades de producción agrícola en los municipios de Calera, General Enrique Estrada, Guadalupe, Morelos y Zacatecas - cuyos territorios cubren la mayor parte de los tres acuíferos mencionados - cuenta con riego. ${ }^{3} \mathrm{El} 8$ o por ciento de ellos obtiene el "oro azul" de pozos profundos; los demás, de pozos a cielo abierto (Io por ciento), de presas (como La Zacatecana, 5 por ciento) y en menor medida de bordos, ollas de agua o directamente de arroyos contaminados. ${ }^{1 / 4}$ Los agricultores locales no tienen que pagar por el agua que se extrae de los mantos freáticos para la irrigación y muy pocos tienen medidores.

En general, sus sistemas de riego exhiben "bajos niveles de tecnificación", lo que quiere decir que se emplean con frecuencia canales de tierra, con grandes pérdidas de agua por la evaporación y la ineficiencia en general. Sólo 6 por ciento de las unidades de producción con superficie de riego en los municipios mencionados cuenta con regadío por goteo. ${ }^{\mathrm{In}}$

En los municipios de Calera, General Enrique Estrada y Morelos, donde se ubica la parte sur del acuífero más sobreexplotado de la región (Calera), la mayor parte de la superficie dedicada a actividades agropecuarias es privada: 76 por ciento, 95 por ciento y 60 por ciento, respectivamente. En cambio, en el municipio de Guadalupe, donde las aguas superficiales y subterráneas están más contaminadas, casi tres cuartas partes de la tierra agrícola es ejidal. ${ }^{16}$ 
Cuadro 1: Distribución sectorial de aguas concesionadas

de los acuiferos que abastecen la ZCZG

\begin{tabular}{|c|c|c|c|c|c|c|c|c|}
\hline & \multicolumn{2}{|c|}{ Benito Juárez } & \multicolumn{2}{|c|}{ Calera } & \multicolumn{2}{|c|}{ Guadalupe-Bañuelos } & \multicolumn{2}{|c|}{ Total } \\
\hline & $\begin{array}{c}\text { Vol. } \\
\text { Mm3/año }\end{array}$ & $\%$ & $\begin{array}{c}\text { Vol. } \\
\text { Mm3/año }\end{array}$ & $\%$ & $\begin{array}{c}\text { Vol. } \\
\text { Mm3/año }\end{array}$ & $\%$ & $\begin{array}{c}\text { Vol. } \\
\text { Mm3/año }\end{array}$ & $\%$ \\
\hline Agrícola & $\mathrm{I} 2.2$ & $57 \cdot 4$ & I2/.6 & 77.3 & 5.4 & 42.6 & $\mathrm{I} / 42.2$ & 72.9 \\
\hline $\begin{array}{l}\text { Público } \\
\text { urbano }\end{array}$ & $9 \cdot 0$ & 42.5 & 22.7 & I $4 . I$ & 7.0 & 55.6 & 38.8 & I9.9 \\
\hline Industrial & o & o & I3.o & $8 . \mathrm{I}$ & o & o & I3.0 & 6.7 \\
\hline Otros* & 0.02 & O.I & 0.8 & 0.5 & 0.24 & І. 8 & I.I & 0.5 \\
\hline Total & $2 \mathrm{I} .2$ & IOO & I6I.o & IOO & 12.7 & IOO & I95.o & IOO \\
\hline
\end{tabular}

Fuente: Elaboración propia con base en el Registro de Usuarios proporcionado por el ingeniero Rafael Guardado, Jefe del Departamento de Aguas Subterráneas de la Dirección Local Zacatecas de la Conagua, en agosto de 2012 .

* Los otros sectores incluyen servicios, doméstico, pecuario y agroindustrial.

\section{INDUSTRIA CERVECERA}

No cabe duda que el acuífero Calera tiene la mejor calidad de agua en la región. Es menos "dura" que el agua extraída de los otros dos acuíferos que abastecen la ZCZG. En otras palabras, no tiene altas concentraciones de calcio y magnesio ("sales"), como las aguas subterráneas de los acuíferos Benito Juárez y Guadalupe Bañuelos, las cuales producen incrustaciones en los conductos que distribuyen el agua en la ZCZG. Además, los estudios citados arriba indican que el agua de Calera es mucho menos contaminada, con concentraciones de flúor, arsénico y otros metales pesados dentro de la Nom para el agua potable (NOM-I27-SSAI-I994). Por tanto, esta agua es la más codiciada de la región.

Como se mencionó anteriormente, desde su inicio la Conagua ha tenido conocimiento del alto grado de sobreexplotación del acuífero Calera. Los estudios técnicos llevados a cabo por dicho organismo en 1992 indican que la recarga natural de Calera es de $83.9 \mathrm{Mm}^{3}$ año y que en aquel entonces se extraía $125 \mathrm{Mm}^{3} /$ año. En 2009, la Conagua elaboró un informe en donde se señala que los volúmenes concesionados suman I5̆..2 $\mathrm{Mm}^{3}$ /año, lo que se traduce en un déficit de $67.75 \mathrm{Mm}^{3} /$ año. ${ }^{17}$ Ahora, con base en el Registro de Usuarios proporcionado por la Conagua en Zacatecas, en 2012 se observa que los volúmenes de las concesiones han alcanzado ı6r.o Mm3/año, subiendo la tasa de sobreexplotación a 77.r Mm³/año. ¿Cómo es posible que el volumen concesionado siga creciendo cuando el acuífero Calera forma parte de una zona de veda desde ig6o?
En este contexto, el caso del Grupo Modelo es notorio. Desde una perspectiva de sustentabilidad ecológica y social, la instalación de una gigantesca fábrica de cerveza en una zona árida y encima de un acuífero ya agudamente sobreexplotado parece una burla. Sin embargo, de acuerdo con la lógica neoliberal y los intereses del gran capital nacional, en I99ı el Grupo Modelo - bajo el mando de Valentín Díez Morodo, quien fungió como presidente del poderoso Consejo Mexicano de Hombres de Negocio - empezó a instalarse en la región. Seis años más tarde se inauguró la planta de la Compañía Cervecera de Zacatecas (una de las siete grandes subsidiarios del Grupo Modelo), en el municipio de Calera, a 3 o km de la capital del estado. Según reportes periodísticos, el Grupo Modelo compró los pozos de los agricultores locales, pagando hasta 200 mil pesos para quedarse con los derechos de usufructo. ${ }^{18}$ Hoy en día, tiene ocho concesiones para extraer $4.85 \mathrm{Mm}^{3} /$ año del acuífero Calera. ${ }^{19}$

En 2013, el Grupo Modelo fue vendido en su totalidad a la empresa belga y brasileña Anheuser-Busch InBev, la mayor fabricante de cerveza en el mundo. Esta gigante empresa transnacional pagó 20.I mil millones de dólares para adquirir el Grupo Modelo, lo que se considera la mayor cantidad de dinero pagada por una empresa mexicana. El 8o\% de este dinero corresponde a un fideicomiso conformado por Valentín Díez Morodo, María Asunción Aramburuzabala y Carlos Fernández González, fieles representantes de la gran burguesía mexicana y también de la clase capitalista transnacional. Ahora, le toca a una empresa transnacional extranjera la oportunidad de minar las aguas 


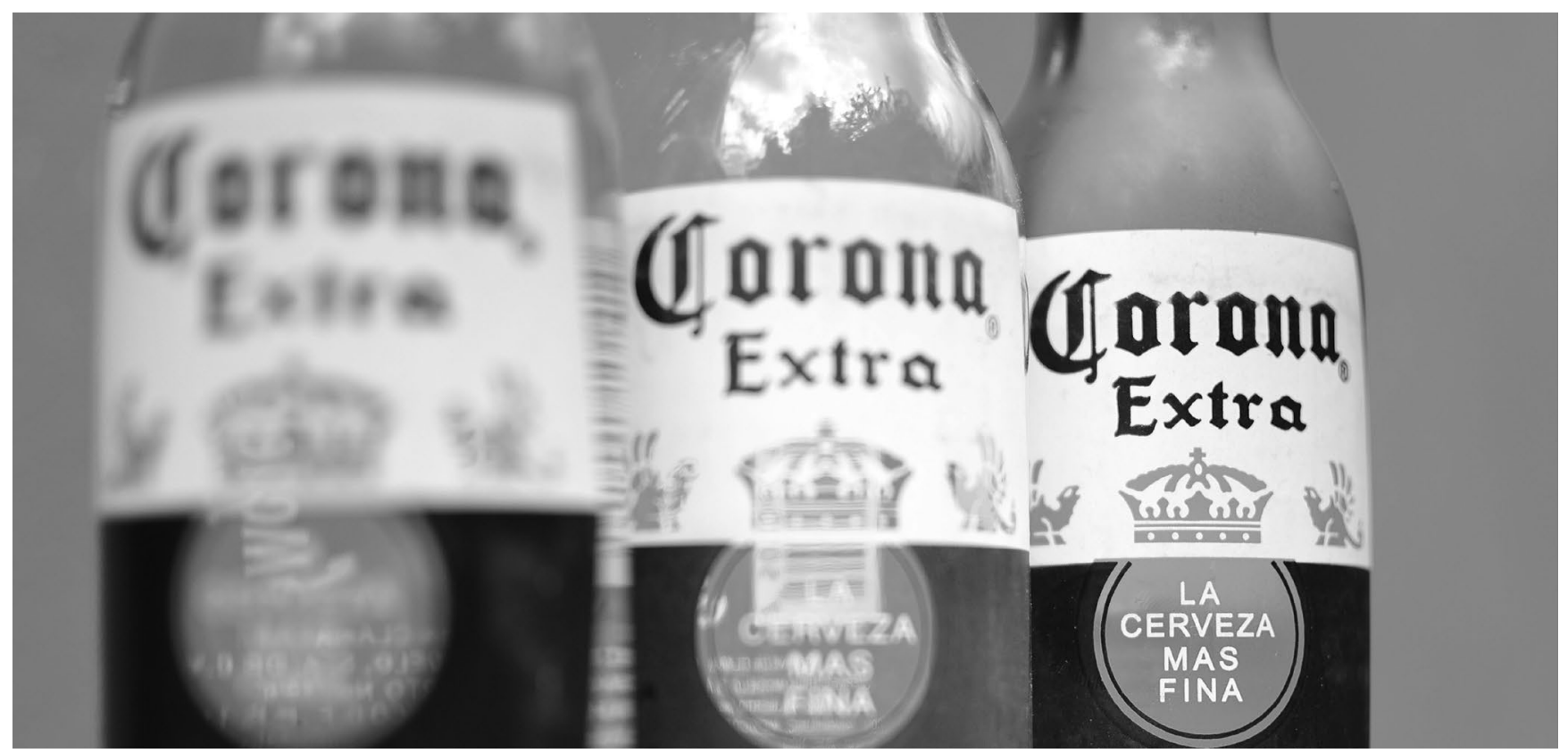

La cerveza zacatecana de mayor exportación, propiedad de una empresa multinacional, es producida con las mejores aguas subterráneas de Calera. El acaparamiento de pozos por la compañía afecta el riego agrícola y el consumo humano.

Foto: Gerardo Márquez

subterráneas de Calera, a un costo nominal de menos de nueve centavos por metro cúbico, según la Ley Federal de Derechos de 2013.

\section{LA SED DE LA CIUDAD}

La ZCZG consume $27 \mathrm{Mm}$ 3/año. Las aportaciones de los tres acuíferos analizados en este texto son relativamente iguales: Benito Juárez provee 34.9 por ciento, Guadalupe-Bañuelos 34.8 por ciento y Calera 30.2 por ciento. ${ }^{20}$ De esta manera, casi 70 por ciento del agua consumida en la ZCZG proviene de los dos acuíferos con aguas "duras", donde se han detectado metales pesados en concentraciones por arriba de la Nom. De acuerdo con los volúmenes asignados por medio de concesiones, el sector público urbano consume 42.5 por ciento de las aguas extraídas de Benito Juárez, el 55.6 por ciento de Guadalupe Bañuelos y sólo I/4.I por ciento de las aguas relativamente limpias de Calera (véase el cuadro i). Esta distribución puede causar sorpresa en vista de la prioridad asignada al consumo público urbano en la Ley de Aguas Nacionales.

La Junta Intermunicipal de Agua Potable y Alcantarillado de Zacatecas (JIAPAZ) es la principal agencia gubernamental responsable de administrar el suminis- tro de agua entubada a la ZCZG. La JIAPAZ es un organismo gubernamental descentralizado que opera en los municipios de Zacatecas, Guadalupe, Morelos y Vetagrande. Presume que sus servicios tienen una cobertura de casi 99 por ciento de las viviendas en su jurisdicción. Sin embargo, los recortes son frecuentes, sobre todo en las colonias marginadas, y en algunos fraccionamientos marginados los pobladores tienen que depender de un servicio irregular de suministro por pipa.

El volumen de agua manejado por la JIAPAZ se traduce en una dotación de 236.6 litros diarios de agua por habitante $(\mathrm{l} / \mathrm{d} / \mathrm{h})$ en los cuatro municipios mencionados. Esta cifra es considerablemente más que el es-

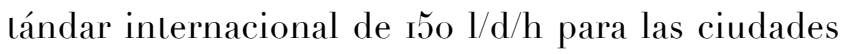
sustentables; y si se compara con el uso de agua en algunas ciudades europeas, donde el consumo per cápita es de alrededor de Ioo $\mathrm{l} / \mathrm{d} / \mathrm{h}$, se pone en evidencia el amplio margen que existe para mejorar la eficiencia del uso de agua en la ZCZG.

Se estima que 40 por ciento del agua suministrada a la ZCZG se pierde en fugas y tomas clandestinas. Hay partes de la infraestructura hidráulica que tienen más de 3 o años de edad, donde el deterioro se traduce en fugas frecuentes. ${ }^{2 I}$ Por otra parte, las incrustaciones de sales restringen el flujo del agua en la tubería de distribución, lo que aumenta los costos de mantenimiento. 


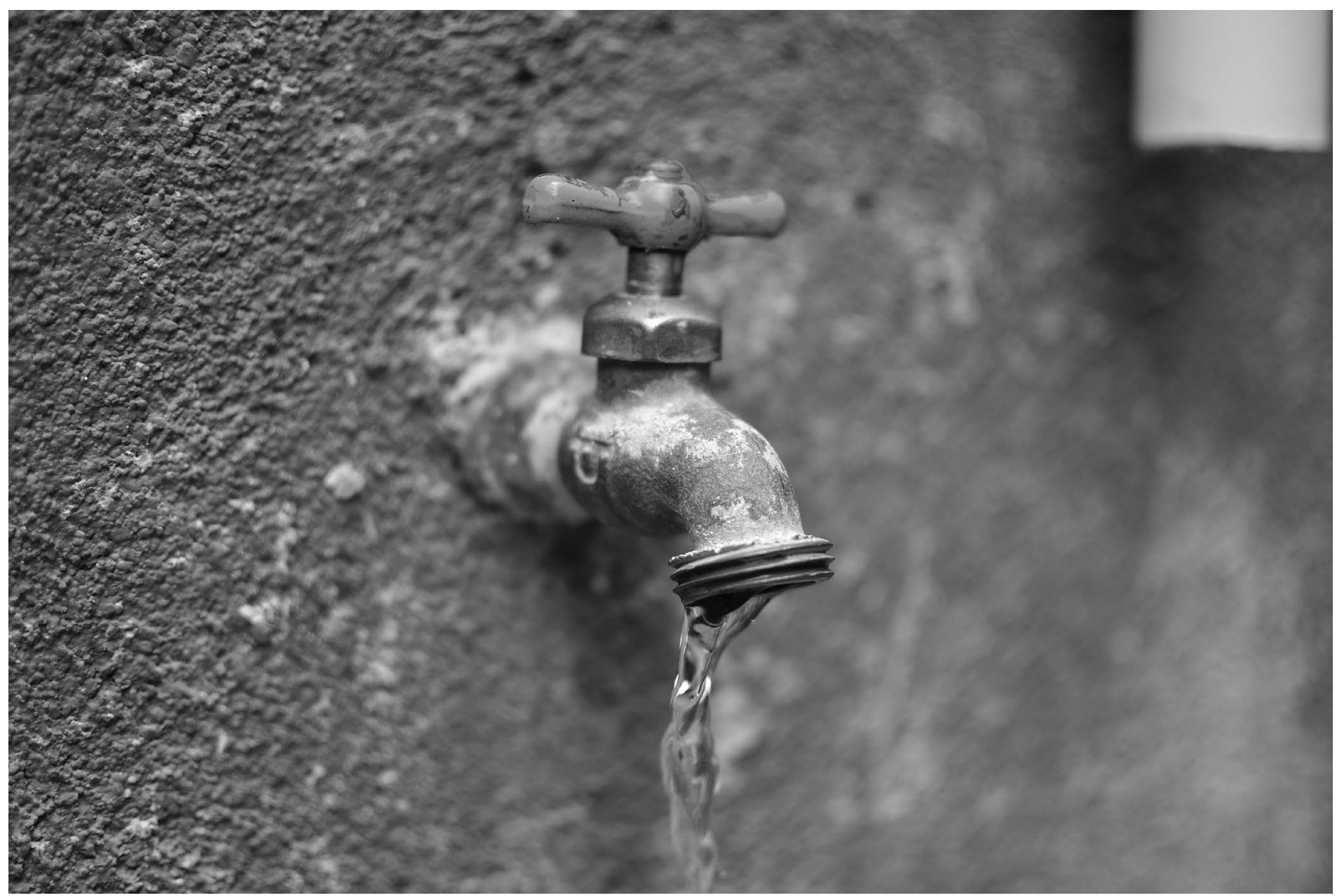

La escasez del agua potable en las tomas domiciliarias de las colonias populares es un problema cotidiano.

Muchos consumidores utilizan este líquido para beberlo y preparar alimentos sin tomar en cuenta que contiene metales pesados y otras sustancias nocivas para la salud.

Foto: Gerardo Márquez

Otro problema es que no hay buena contabilidad. De un total de 92,7I 4 usuarios de la JIAPAZ, II,280 tienen una "cuota fija"; en otras palabras, no tienen un medidor y pagan el precio del consumo mínimo sin importar la cantidad de agua que consumen. Adicionalmente, hay 5,I82 usuarios en la ciudad de Zacatecas que tienen medidores sin lectura o sin funcionar. En estos casos también el usuario paga el precio del consumo mínimo, sin importar la cantidad real de consumo. ${ }^{22}$

Por último, pero no por eso menos importante, los habitantes de la ZCZG no están conscientes de la crisis de agua que se perfila en la región. En 20I3, investigadores de la Universidad Autónoma de Zacatecas (UAZ) aplicaron una encuesta a 242 hogares en la ZCZG para detectar la percepción de la gente sobre la problemática del agua. La encuesta fue aplicada en las colonias Arboledas (54), Centro de Zacatecas (59), Centro de Guadalupe $(57)$, Las Huertas (33) y Lomas Bizantinas (39).

Nos sorprendió aprender que $7^{3}$ por ciento de los encuestados ni siquiera sabe de dónde viene el agua que consume: ¿de un río?, ¿una presa?, ¿un pozo? No se sabe. Y entre los pocos que sí saben, dos terceras partes reporta no tener conocimiento sobre los problemas de sobrexplotación y contaminación. Paradójicamente, más de go por ciento de los encuestados reportan tomar medidas para ahorrar el agua, siendo las más comunes el reciclaje de agua, no dejar que corra de la llave sin necesidad y poner un bote en la regadera mientras que se baña. Aunque sean medidas pocas efectivas para reducir sensiblemente el consumo de agua, el reportar sobre ellas sugiere que los pobladores de la ZCZG sí valorizan el líquido vital.

\section{Conclusiones y REFLEXIONES FINALES}

La problemática del agua en la ZCZG constituye una crisis y un conflicto socioambiental latente. Los acuíferos están sobreexplotados. Se han detectado concentraciones de metales pesados por arriba de la NOM en el agua 
destinada al consumo público urbano. El agua de mejor calidad se entrega a las grandes empresas (trans)nacionales y a los agricultores privados, mientras que las aguas "duras" y más contaminadas de los acuíferos Benito Juárez y Guadalupe-Bañuelos se destinan al sector social del campo y al sector público urbano.

Al cumplir 25 años de vida, la Conagua no ha logrado reducir las tasas de sobreexplotación de los acuíferos que abastecen la ZCZG; mantiene vigentes centenares de concesiones de explotación que en su conjunto representan volúmenes de agua mayores que los disponibles; no ha obligado a los usuarios a instalar medidores, de acuerdo con la ley; y ha mostrado disposición a privilegiar los intereses del gran capital.

Por cierto, esto no quiere decir que todos los programas de la Conagua son malos de por sí. Por ejemplo, un programa denominado Manejo Sustentable de Recursos Hídricos en el Estado de Zacatecas comprende las siguientes líneas de acción: mejorar la reglamentación; reconversión productiva mediante la introducción de canola, un cultivo que utiliza menos agua que los cultivos tradicionales de la región; reconversión productiva mediante la introducción de nopal forrajero, otro cultivo que consume relativamente poca agua; introducción de riego por goteo para chile seco y alfalfa, la construcción de macro-túneles para irrigar chile verde; adecuación de derechos de riego; y el uso de aguas residuales tratadas para la irrigación. El punto es que estos programas están subordinados a los intereses del capital, además de ser pobremente financiados. En 2013, el presupuesto de la Conagua a nivel nacional (4r.5 mil millones de pesos) sólo representó I.36 por ciento del gasto programable.

El agua limpia fluye hacia el dinero. Las grandes empresas nacionales y transnacionales compran el agua subterránea en Zacatecas a precios relativamente bajos para llevar a cabo sus actividades económicas, orientadas a maximizar las ganancias de sus principales accionistas. De esta manera, se permitió que el Grupo Modelo se instalara en Calera para tomar una gran mordida del ya súper-sobreexplotado acuífero Calera. Asimismo, en años más recientes la Conagua ha otorgado a la compañía canadiense Goldcorp el derecho de sobreexplotar el acuífero Cedros, en el municipio de Mazapil, a una tasa cuatro veces mayor que su recarga natural, lo que priva a los campesinos locales del agua que ellos necesitan para irrigar sus cultivos. ${ }^{23}$

Inversamente, el agua contaminada fluye hacia el sector social del campo y los pobladores del zczG. En esta dirección, las aguas negras no tratadas pasan por las colonias marginadas en la periferia de la ZCZG y siguen su cauce hasta las tierras ejidales en el municipio de Guadalupe. Asimismo, los residuos tóxicos de más de 400 años de explotación minera capitalista se han dispersado por todas partes del valle de Guadalupe, concentrándose en la presa La Zacatecana. Con el tiempo, estos desechos han infiltrado a los acuíferos Benito Juárez y Guadalupe-Bañuelos, donde se ha detectado la presencia de metales pesados por encima de la Nom. Nuevamente, estas son las aguas que abastecen 70 por ciento de la demanda de agua en la ZCZG.

El Banco Mundial aboga por más mercantilización y privatización del agua en la ZCZG. En un estudio publicado en 2012, pone en el centro de su propuesta aumentar la oferta de agua en el sector agrícola a través de la construcción de bordos y el reciclaje de aguas tratadas; construir plantas potabilizadoras para tratar las aguas destinadas el sector público urbano; aumentar la recaudación de los pagos por los servicios de agua; y "establecer condiciones que permitan a la iniciativa privada participar en la prestación de servicios". ${ }^{24}$ Sobre esta línea, el Banco prevé la necesidad de liquidar trabajadores de la JIAPAz.

Desde nuestra perspectiva, esta es una receta para agudizar el conflicto socioambiental en torno al agua en Zacatecas. Cuanto más se mercantilice el agua, más fácilmente fluye hacia el poder económico, exacerbando así la mala distribución del líquido vital. Al seguir el camino neoliberal, se advierten mayores niveles de conflictividad, conforme la crisis se manifiesta cada vez más en problemas de abastecimiento, contaminación, afectaciones a la salud y productividad.

Hasta ahora, el conflicto ha estado "latente", pues, en general, los pobladores de la ZCZG no están conscientes de la crisis de agua. Esto se explica en gran medida por el hecho de que el agua subterránea no se ve, tampoco la contaminación por metales pesados. Sin embargo, los niveles piezométricos de los acuíferos se abaten entre cinco y ı m cada año; el cambio climático probablemente se traducirá en sequías cada vez más frecuentes e intensas (como la de 20II y 20I2); y el aumento en la escala y ritmo de las actividades mineras en el marco neoliberal promete mayores niveles de contaminación tóxica en el futuro.

¿Cuáles son las alternativas? En primer lugar, hay que insistir en que el acceso al agua limpia es un derecho humano universal, tal como se señala en el Artículo 4 de la Constitución mexicana y también en el Pacto Internacional de Derechos Económicos, Sociales y Culturales. Desde esta perspectiva, el Estado tiene la obligación de 


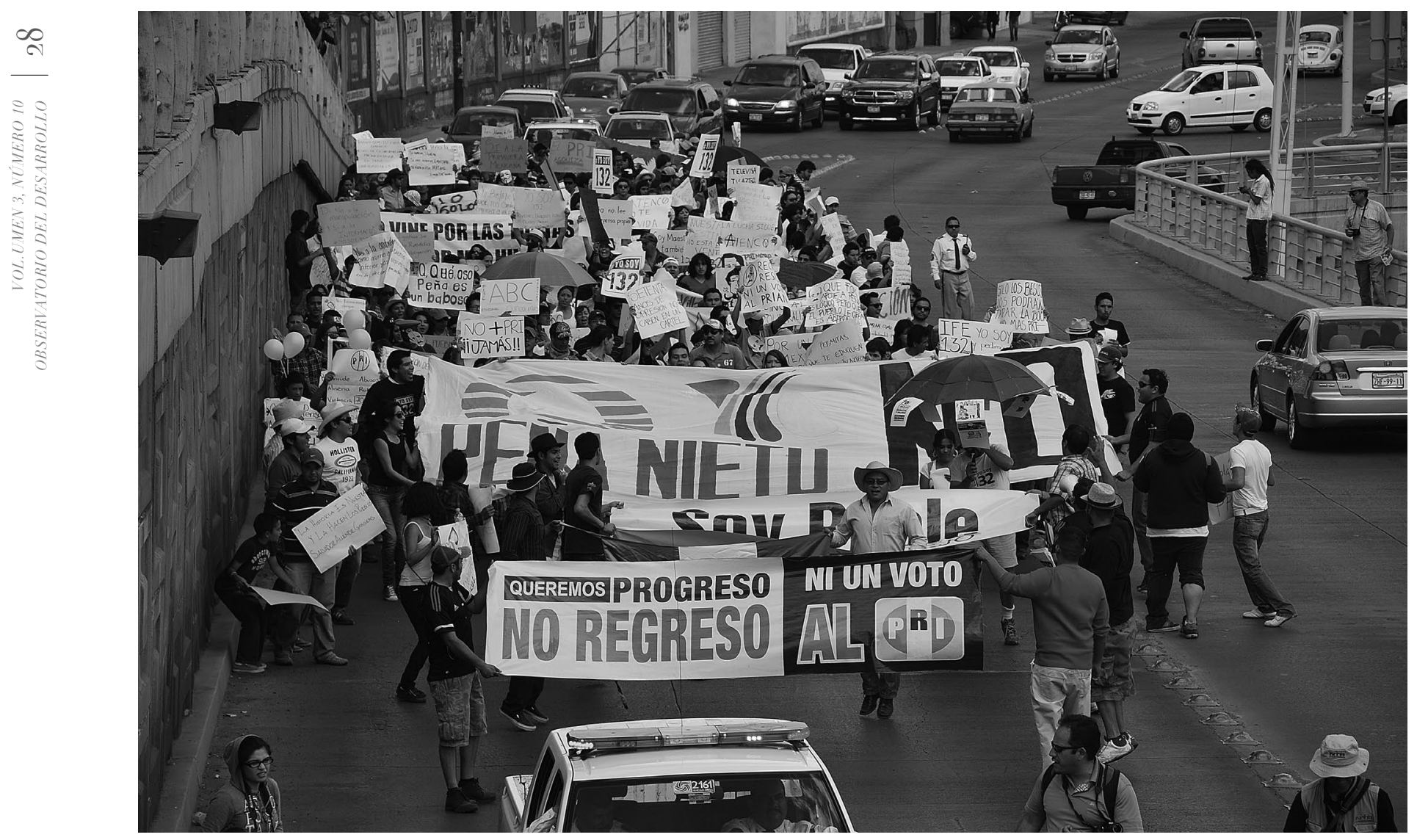

Diversos sectores de la sociedad, entre ellos los jóvenes, se han manifestado en contra de la imposición mediática en la presidencia de la República. Tentativamente, pudieran abrazar causas fundamentales

como el derecho al agua limpia, gratuita y suficiente.

Foto: Jorge VÁzquez.

asegurar que todos los ciudadanos tengan acceso a suficiente agua potable para satisfacer las necesidades básicas. Pedro Arrojo sugiere que los primeros 3 o a $40 \mathrm{ol} / \mathrm{d} / \mathrm{h}$ sean abastecidos en forma gratuita, por lo menos para los hogares más pobres, para garantizar el derecho humano al agua. ${ }^{25}$ De ahí, se recomienda manejar un sistema tarifario escalonado para desincentivar el derroche y para obligar a los grandes consumidores a pagar caro.

De manera complementaria, Peter Gleik apunta al "camino suave del agua" para incorporar valores ecológicos en las políticas públicas relacionadas, enfocarse en satisfacer necesidades humanas básicas y romper el vínculo entre la acumulación de capital y el uso del agua. ${ }^{26}$ Desde este paradigma, se aboga por mayores niveles de participación de la sociedad civil en la vigilancia de los organismos públicos y en la búsqueda de soluciones integrales y multifacéticas. Se pone énfasis en la conservación, eficiencia y el reúso del agua; en la captación de aguas pluviales y la reclamación de aguas tratadas, según las posibilidades hidrológicas, técnicas y culturales de la región.
En último análisis, la incorporación de una racionalidad ecológica y social en el manejo del agua y de otros bienes comunes requiere romper con el modelo neoliberal; necesita la confluencia de movimientos sociales comprometidos en reconfigurar las políticas públicas alrededor de los principios de igualdad, solidaridad, sustentabilidad fuerte, diversidad biocultural, permacultura, agroecología, soberanía alimentaria y autonomía local; y demanda una revalorización del agua como el líquido vital de la vida (valor de uso), en lugar de tratarla como si fuera una mercancía (valor crematístico). Sobre este camino revolucionario, la concientización, politización y auto-organización de los ciudadanos son condiciones sine qua non. En la ZCZG, las organizaciones de base popular en el campo (como El Barzón), los movimientos estudiantiles (capítulos locales del zapatismo y del movimiento YoSoyı32) y también el Movimiento Mayo 29, que agrupa activistas sociales desde hace 40 años, están en la vanguardia de las luchas sociales. Es hora de tomar la bandera del agua. 
'Secretaría de Agricultura y Recursos Hidráulicos (SARH) (1988), Actualización geohidrológica en la zona de Guadalupe Bañuelos, para suministro de agua a la ciudad de Zacatecas, Zac., México, SARH, p. I.

${ }^{2}$ Misión de la Conagua, citada de su sitio de Internet (http:/ www.conagua.gob.mx).

${ }^{3}$ Cifras citadas de una serie de reportes de la Conagua, titulados Actualización de la disponibilidad media anual de agua subterránea, para los acuíferos Calera, Benito Juárez y Guadalupe-Bañuelos, disponibles en su sitio de Internet.

IIdem.

${ }^{5}$ Cálculos propios, con base en el Registro de Usuarios proporcionado por el ingeniero Rafael Guardado, Jefe del Departamento de Aguas Subterráneas de la Dirección Local Zacatecas de la Conagua, en agosto de 2012.

${ }^{6}$ Elvira Santos-Santos, Mario Yarto-Ramírez, Irma Gavilán-García, et al. (2006), "Analysis of Arsenic, Lead and Mercury in Farming Areas with Mining Contaminated Soils at Zacatecas, Mexico", Journal of Mexican Chemical Society, vol. 5o, no. 2 , pp. $57-63$

7Gobierno del Estado de Zacatecas, Secretaría del Medio Ambiente y Recursos Naturales (Semarnat) et al. (2002), Plan de acción de la presa la zacatecana para la contención de metales pesados municipio, http://www.inecc.gob.mx/descargas/sqre/zacatecana. pdf, p. 37 .

${ }^{8}$ Comisión Nacional del Agua (Conagua) (I998), "Estudio para evaluar la contaminación de los acuíferos donde están ubicadas las fuentes de abastecimiento para agua potable de las ciudades de Zacatecas, Guadalupe y poblaciones circunvecinas", Conagua, Subgerencia Técnica, Gerencia Estatal Zacatecas, Departamento de Aguas Subterráneas, Guadalupe, Zac., p. 9 y p. 26.

${ }_{9}$ Nelly Molina Frechero, Leonor Sánchez Pérez, Enrique Castañeda Castaneira, et al. (2013), "Drinking Water Fluoride Levels for a City in Northern Mexico (Durango) Determined Using a Direct Electrochemical Method and Their Potential Effects on Oral Health," The Scientific World Journal, vol. 2013, Article ID I86392.

'o Takahiko Yoshida, Hiroshi Yamauchi y Gui Gan Sun (2004), "Chronic health effects in people exposed to arsenic via the drinking water: dose-response relationships in review", Toxicology and Applied Pharmacology, vol. 198, no. I, pp. 2/3-252.

"http:/www.lasnoticiasya.com/2012/12/o8/en-ceapa-muchas-obras-y-poca-claridad/

${ }^{12}$ Conagua (1998), op. cit., p. 8.

${ }^{13}$ Cálculos propios, basados en datos obtenidos de Instituto Nacional de Estadística, Geografía e Informática (INEGI) (2007), VIII Censo agricola, ganadero y forestal 2007, http://www.inegi.org.mx/ sistemas/TabuladosBasicos/Default.aspx? $\mathrm{c}=17 \mathrm{7} 7 \mathrm{7} \& \mathrm{~s}=\mathrm{est}$

4Idem.

"5. Idem.

${ }^{16}$ Idem.

${ }^{7}$ Comisión Nacional de Agua (Conagua) (2009), Actualización de la disponibilidad media anual de agua subterránea, acuífero (3225) Calera, estado de Zacatecas, Subdirección General Técnica, Gerencia de Aguas Subterráneas, México.

${ }^{18}$ Rosa Rojas (2012), "Zacatecas tardará una década en sanar de la sequía", La Jornada, Jueves, 2I de junio, p. 2.

${ }^{19}$ Cálculos propios, con base en el Registro de Usuarios 2012, op. cit.

${ }^{\circ}$ Darcy Tetreault y Cindy McCulligh (2013), "El camino suave del agua. Una alternativa para superar la crisis en la zona conurbada de Zacatecas y Guadalupe", Observatorio del Desarrollo, vol. 2, no. 4, pp. 33-37.

"César Pérez (200I), "Derecho al agua, un bien natural que sólo algunos pueden costear", La Jornada de Zacatecas, Domingo, II de Diciembre, p. 2.

${ }^{22}$ Tetreault y McCulligh (20I3), op. cit.

${ }^{23}$ Claudio Garibay, Andrés Boni, Francisco Panico, et al. (2011), "Unequal Partners, Unequal Exchange: Goldcorp, the Mexican State, and Campesino Dispossession at the Peñasquito Goldmine", Journal of Latin American Geography, vol. ı, no. 2, pp. I53-176.

${ }^{2}$ Banco Mundial (2012), Análisis del uso y manejo de los recursos hídricos en el estado de Zacatecas, Washington, Banco Mundial, p.67.

${ }^{25}$ Pedro Arrojo, (2009), "El reto ético de la crisis global del agua”, Relaciones Internacionales, no. I2, pp. 33-53.

${ }^{26}$ Peter Gleik (2000), "The Changing Water Paradigm: A Look at Twenty-first Century Water Resources Development", Water International, vol. 25, no. I, pp. 127-138. 
Unidad Académica de Estudios del Desarrollo

Red internacional de Desarrollo, Sustentabilidad y Seguridad Humana

A través del Seminario Permanente del Desarrollo

Presenta la conferencia:

\section{UNA RETROSPECTIVA SOBRE LOS EFECTOS DEL TLCAN EN MÉXICO 1994-2014} de James M. Cypher

Comenta: Roberto Soto Esquivel, UAED-UAZ

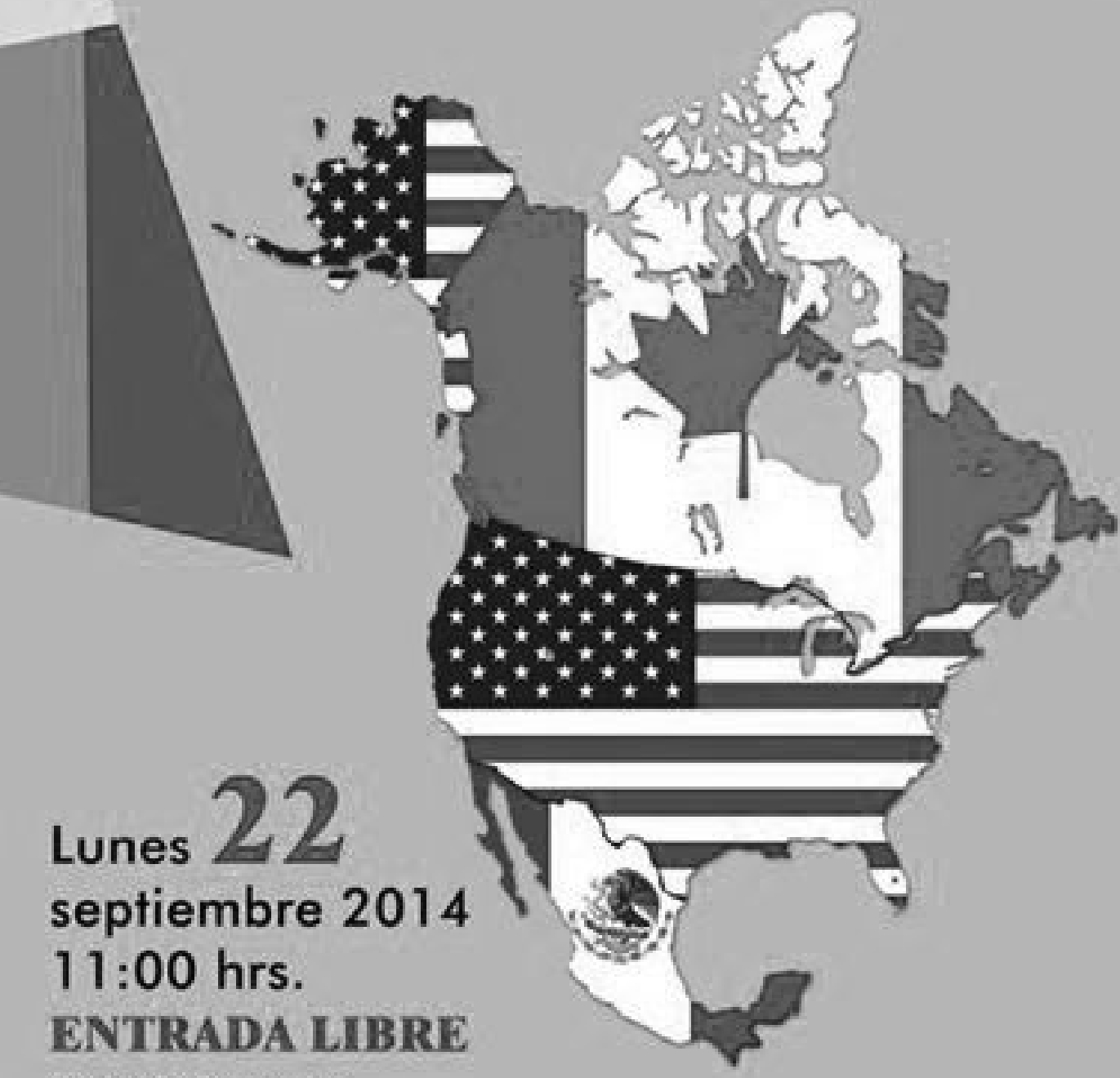

Salón de Doctorado

Campus Universitario II

Av. Preparatoria $s / n$

Col. Hidráulica

Zacatecas, Zac.

TeL. (492) 8996945

www.estudiosdeldesarrollo.net 